\title{
Protein Folding Sculpting Evolutionary Change
}

\author{
S. LINDQUIST \\ Whitehead Institute for Biomedical Research, Massachusetts Institute of Technology, \\ Cambridge, Massachusetts 02142 \\ Correspondence: lindquist_admin@wi.mit.edu
}

\begin{abstract}
Our work suggests that the forces that govern protein folding exert a profound effect on how genotypes are translated into phenotypes and that this in turn has strong effects on evolutionary processes. Molecular chaperones, also known as "heat-shock proteins" (Hsps), promote the correct folding and maturation of many other proteins in the cell. Hsp90 is an abundant and highly specialized chaperone that works on a particularly interesting group of client proteins: metastable signal transducers that are key regulators of a broad spectrum of biological processes. Such proteins often have evolved to finish folding only when they have received a specific signal, such as the binding of a ligand or a posttranslational modification. Importantly, the folding of Hsp90 clients is particularly sensitive to changes in the external and internal environment of the cell. Therefore, Hsp90 is uniquely positioned to couple environmental contingencies to the evolution of new traits. Our work has helped to define two mechanisms by which Hsp90 might influence the acquisition of new phenotypes. First, by robustly maintaining signaling pathways, Hsp90 can buffer the effects of mutations in those pathways, allowing the storage of cryptic genetic variation that is released by stress. In this case, when the Hsp90 buffer is compromised by environmental stress, new traits appear. These traits can also be assimilated, so that they become manifest even in the absence of stress, when genetic recombination and selection enrich causative variants in subsequent generations. Second, $\mathrm{Hsp} 90$ can potentiate the effects of genetic variation, allowing new mutations to produce immediate phenotypes. In this case, when Hsp90 function is compromised, new traits are lost. These traits can also be assimilated, so that they are maintained under environmental stress, but this is achieved through new mutations. We have discovered these powerful evolutionary mechanisms in fruit flies, mustard plants, and fungi, but expect them to operate in all eukaryotes. Another line of work relating protein folding to the evolution of new traits involves protein-based hereditary elements known as prions. These produce changes in phenotype through heritable, self-perpetuating changes in protein conformation. Because changes in protein homeostasis occur with environmental stress, prions can be cured or induced by stress, creating heritable new phenotypes that depend on the genetic variation present in the organism. Both prions and Hsp90 provide plausible mechanisms for allowing genetic diversity and fluctuating environments to fuel the pace of evolutionary change. The multiple mechanisms by which protein folding can influence the evolution of new traits provide both a new paradigm for understanding rapid, stepwise evolution and a framework for targeted therapeutic interventions.
\end{abstract}

Proteins are synthesized as long, linear chains of amino acids. To function, they must fold into intricate, stereotyped, three-dimensional structures. Moreover, individual proteins must do so in a highly chaotic environment. Inside a living cell, the protein concentration reaches $300 \mathrm{mg} / \mathrm{ml}$, and those many proteins possess a tremendous amount of kinetic energy. Individual proteins therefore frequently encounter folding problems due to macromolecular crowding. In fact, "off-pathway" folding events are a constant problem. Individual cells, and even entire organisms, live poised on the precipice of a protein-folding crisis, a situation that is worsened by environmental stress.

In the last 15 years, two different areas of research in my laboratory have pointed to a pivotal role for protein folding in evolutionary processes. One of these involves the action of chaperone proteins, which facilitate protein folding in the complex cellular environment, thereby helping proteins to assume their normal functions. The second closely related line of work involves a type of hereditary element, a prion, that produces a change in phenotype through a heritable, self-perpetuating change in protein conformation. Reflecting time constraints of the Symposium, I focus my presentation on the role of the chaperone protein Hsp90 and only touch on yeast prions very briefly.

\section{HEAT-SHOCK PROTEINS}

Nearly $5 \%$ of the protein mass of the cell is devoted to helping other proteins fold properly and to correcting problems that occur when they misfold. An important class of helper proteins (known as chaperones) is the heatshock proteins, so called because they can be induced by a shift from a normal to a slightly higher growth temperature. We concentrate here on the abundant chaperone Hsp90 and its role in facilitating the rapid evolution of novel traits. Hsp90 is essential for life in eukaryotic cells. Only small amounts of Hsp90 are required for growth and development under normal conditions, but much more is required under stressful conditions. Unlike most chaperones, it is made at much higher levels than needed and is present in excess under normal conditions and therefore acts as a protein-folding buffer. This enables it to facilitate protein folding even during sudden, unexpected stresses.

Also unlike most other Hsps, Hsp90 is involved in the folding of specific regulatory proteins positioned strategically in major signal transduction networks, comprising 
perhaps only $\sim 2 \%-5 \%$ of the total protein in the cell. Hsp90's client proteins are characteristically metastable proteins that do not finish folding into their final stable conformations until something else has activated them, for example, the binding of a ligand, a phosphorylation event, or translocation of the protein to the membrane. Protein complexes of Hsp90 with its clients are therefore extremely dynamic and strongly affected by stress. Their metastable nature means that much higher concentrations of Hsp90 are then required to drive its client proteins into appropriate complexes. Stressful conditions also cause additional cellular proteins to become unfolded (thereby creating increased need for Hsp90 and further depleting the buffer). Together, these properties of Hsp90-client interactions have a strong influence on how genetic variation is translated into phenotypic novelty and diversity.

\section{HSP90 BUFFERS POLYMORPHISMS}

Hsp90 may affect evolutionary change by buffering genetic polymorphisms. The excess Hsp90 chaperone capacity allows a variety of different polymorphisms throughout the genome to accumulate without phenotypic consequence. Within a certain range, it keeps these polymorphisms silent because it is able to maintain the normal folding and functioning of the signal transduction pathways controlled by its client proteins. But when stress depletes the Hsp90 buffer, phenotypes associated with these accumulated polymorphisms are revealed suddenly and in a combinatorial fashion. In this way, Hsp90 acts as a "capacitor," allowing the storage and controlled release of genetic variation. This can have enormous functional consequences for the organism.

Our first experiments suggesting this mechanism involved some Hsp90 mutants in Drosophila lines that we obtained from the Rubin and Hoffmann laboratories (Rutherford and Lindquist 1998). We confirmed their findings that homozygotes (completely lacking Hsp90 function) died, and heterozygotes (with reduced Hsp90 function) were normal. However, we noticed that a small number of them had astonishing, and diverse, developmental anomalies (Fig. 1A-F). Interestingly, these rare developmental anomalies were generally specific to the genetic background into which the mutations were transferred. These observations suggested that reducing Hsp90 function does not just destabilize development; instead, it reveals the effects of previously hidden genetic variation that can affect virtually every trait in the fly.

Further experiments suggested that populations of these flies contained a great diversity of different genetic polymorphisms. Some rare individuals exhibited several novel and unusual traits. This itself was a big surprise, because most geneticists believed that evolution worked through small incremental mutations. When we reduced Hsp90 function by genetic mutation, or grew flies at a higher temperature, we observed novel phenotypes in those flies more frequently. When we crossed two of the rare flies together and selected for that novel phenotype, we enriched subsequent generations for that underlying genetic variation to the point where those phenotypes were maintained, i.e., variation was assimilated, even when Hsp90 function was normal (Rutherford and Lindquist 1998). We hypothesized that this interface between environmental stress and protein homeostasis could have a large effect on evolution.

We then turned to the mustard plant Arabidopsis thaliana, an inbreeding organism with a sessile lifestyle and considerably less heterozygosity than D. melanogaster. We asked, again, what happened when Hsp90 function was reduced in different accession lines of Arabidopsis. We observed a stunning array of phenotypic abnormalities that were specific to the individual lines: For example, leaves coming out in whorl shapes, plants growing upside down, hairy roots, or vine-like growth (Fig. 2). We also obtained the same array of phenotypes by growing the plants at elevated temperatures (Queitsch et al. 2002; Sangster et al. 2007). Again, these experiments revealed hidden genetic variation when the Hsp90 buffer was reduced by stress.

We next examined the progeny of recombinant inbred plants from different ecotypes. We studied a wide variety of normal life history traits in Arabidopsis, such as flowering time and number of seeds that were set. Every single trait we studied in these lines had some hidden genetic variation that was exposed when Hsp90 function was reduced, and we were able to build a crude map of quantitative trait loci
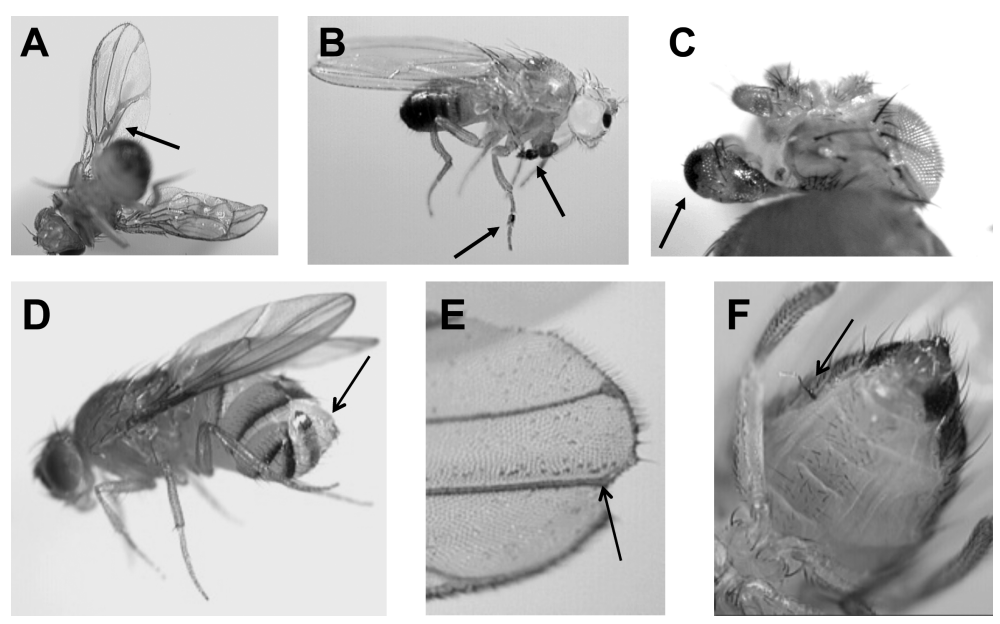

Figure 1. Examples of developmental abnormalities associated with Hsp90 deficits in Drosophila. (A) Thickened wing veins, $(B)$ transformed second leg with an ectopic sex comb, $(C)$ deformed eye with an extra antenna, $(D)$ disorganized abdominal tergites, $(E)$ notched wings, $(F)$ extraneous tissue growing out of tracheal pit. (Reprinted, with permission, from Rutherford and Lindquist 1998 [C Nature Publishing Group].) 

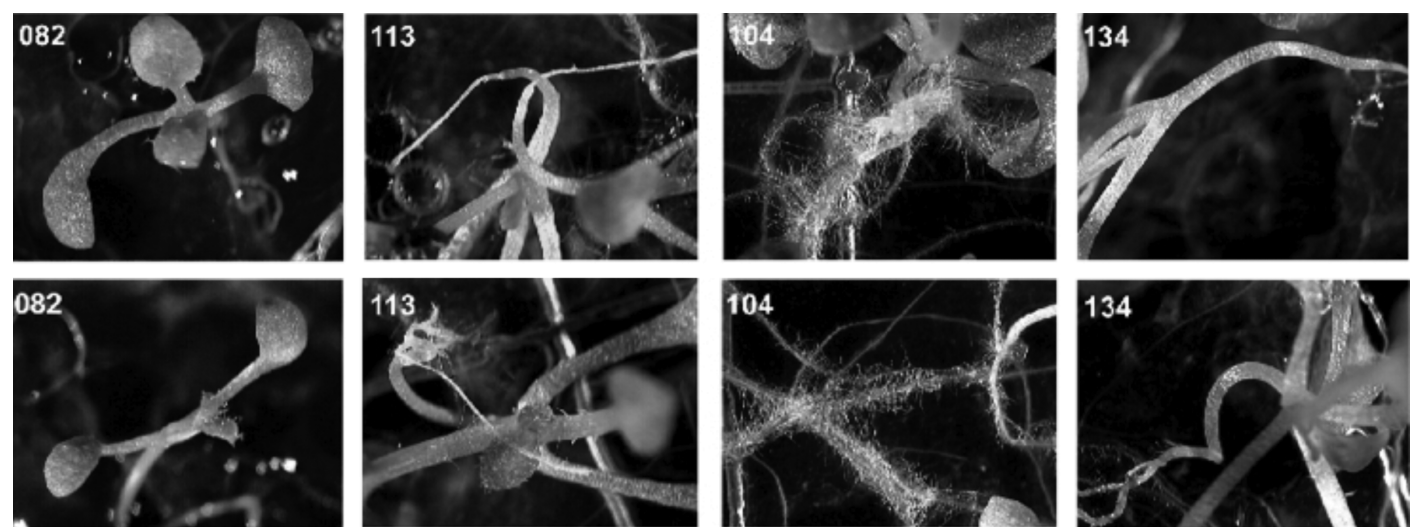

Figure 2. The same recombinant inbred line (RIL)-specific phenotypes are uncovered when Hsp90 buffering capacity is challenged by GdA (top) or by growth at $27^{\circ} \mathrm{C}$ (bottom). (Line 082 seedlings) S-shaped rosettes with vertically oriented leaf blades. (Line 113 seedlings) Extreme hypocotyl curls and roots partially extended into air. (Line 104 seedlings) Abundant root hair growth. (Line 134 seedlings) Bent hypocotyls with rosette touching the medium surface. (Reprinted, with permission, from Queitsch et al. 2002 [C Nature Publishing Group].)

(QTL) involved in these traits by linkage analysis (Sangster et al. 2008a,b). This suggested that most of the underlying variation was genetic rather than epigenetic. In addition to examining Hsp90 inhibition with chemical compounds, we used RNA interference (RNAi) inhibition, establishing that the changes in phenotype were absolutely due to the interface between Hsp90 and genetic variation. That Hsp90 has such a massive influence on the genotype-to-phenotype map in organisms with profoundly different lifestyles, separated by at least 1 billion years of evolution, suggests that this mechanism has influenced evolutionary processes very broadly.

\section{HSP90 POTENTIATES POLYMORPHISMS}

Another way that Hsp90 translates genetic variation into new phenotypes is by directly chaperoning mutated proteins. Some mutations can create new activities in proteins, but often the mutated proteins are unable to fold well on their own. They therefore benefit from the Hsp90 buffer present in the cell to enable them to fold correctly into a functional state and create new phenotypes. In this way, Hsp90 acts as a potentiator of new variation rather than as a capacitor.

The first class of proteins in which we observed this phenomenon was the oncogenic kinases such as v-Src (other kinases have the same basic phenomena). Early experiments on Hsp90 were aimed at determining its basic function and that of the other heat-shock proteins. At that time, we had recently developed techniques to reduce Hsp90 levels in yeast, and we introduced v-Src into those cells to model Hsp90's effects on the protein. Several different laboratories had found that oncogenic kinases were inactive when complexed with Hsp90, leading to speculation that it was a "protein repressor." We found small differences in levels of v-Src accumulation in cells with high versus low levels of Hsp90, but the activity of v-Src in the two cell types was profoundly different (Fig. 3A) (Xu and Lindquist 1993). Surprisingly, rather than being repressed, $\mathrm{v}$-Src activity was much higher in the cells with high lev- els of Hsp90. In contrast, cells with low levels of Hsp90 were able to grow normally but unable to functionally mature the mutated, dysregulated $\mathrm{v}-\mathrm{Src}$ kinase $(\mathrm{Xu}$ et al. 1999). We also found that Hsp90 levels do not make nearly as much difference to the activity of c-Src, a nonmutated and much less active form of the kinase (Fig. 3B). This observation has held true for a host of oncogenic kinases and has been extended to several other types of oncogenes as well. Indeed, studies are under way that suggest that reducing Hsp90 function may offer great promise as a novel therapeutic strategy for treating cancer (Dai and Whitesell 2005; Whitesell and Lindquist 2005; Cullinan and Whitesell 2006).

In any case, these studies suggested that the excess chaperone activity of Hsp90 is a latent reservoir that can be used to fold newly mutated proteins and provide new functions. If such mutations are ever beneficial to the organism, they too could enhance the rate of evolution.

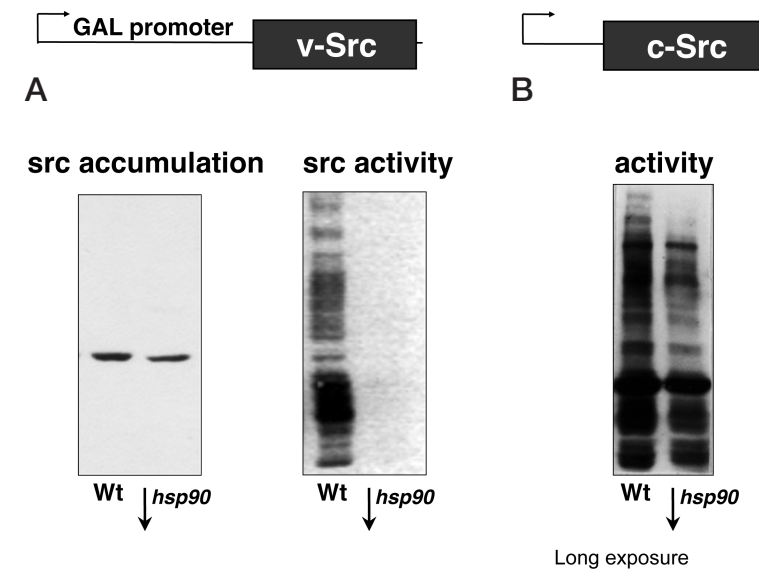

Figure 3. Maturation and activity of the mutated tyrosine kinase v-Src depend on hsp90. (A) v-Src accumulates at the same rate when $h s p 90$ is inhibited, but its activity is reduced. $(B) \mathrm{c}-\mathrm{Src}$ is much less active in general than $\mathrm{v}-\mathrm{Src}$, and its activity is much less dependent on hsp90 levels. 


\section{HSP90 CHAPERONES RESPONDERS}

A related way that Hsp90 affects evolutionary novelty is by chaperoning proteins that respond to mutations. Mutations that confer selective advantages in a new environment might not be advantageous overall because they can simultaneously create other problems. However, the abundant reservoir of Hsp90 can solve this dilemma because it chaperones a host of signal transducers that ameliorate negative effects arising from the mutated protein. We first observed this phenomenon in the evolution of antifungal drug resistance. The yeast Saccharomyces cerevisiae is very adept at evolving resistance when treated with fluconazole, a widely used triazole antifungal agent. But, if Hsp90 activity is reduced pharmacologically or genetically in these cells, their ability to evolve resistance is greatly reduced (Cowen and Lindquist 2005). In this case, Hsp90 was not folding the mutant protein. The mutation was in Erg3, which binds the azole, and the mutations simply eliminated the protein. But Erg3 is involved in sterol synthesis. When it is missing, cells would normally die. They survive only because the reservoir of Hsp90 is available to direct massive maturation of calcineurin (an Hsp90 client) (Imai and Yahara 2000; Cruz et al. 2002; Fox and Heitman 2002; Cowen and Lindquist 2005), which drives many protective changes.

We then tested our hypothesis in a real-time evolution experiment. We obtained a series of Candida albicans isolates from immunocompromised human patients over the course of 2 years of treatment (gift of Ted White, Spencer Redding, and colleagues). Their genetic analysis had revealed that these patients were not being reinfected with a new strain of the pathogen. Rather, a single strain that had already infected them simply kept reblooming. We analyzed these in vivo isolates and we found that even the first isolates were resistant to fluconazole, in comparison to our laboratory strain, and that the level of resistance increased over time. When we added small amounts of geldanamycin (GdA), an Hsp90 inhibitor, we found that it largely eliminated the isolates' fluconazole resistance (Fig. 4) (Cowen and Lindquist 2005), i.e., in this natural evolution setting, all of the mutant cells that had acquired drug resistances depended on Hsp90 for survival.

We observed that in late stages of infection, some of the isolates had also evolved resistance to fluconazole that was maintained even with GdA. But these fungi had never been exposed to Hsp90 inhibitors in the patient. What drove the assimilation of the trait? New mutations that made resistance independent of Hsp90? We hypothesize that this is due to fever, because even a simple increase in growing temperature to $39^{\circ} \mathrm{C}$ phenocopied the specific effects of Hsp90 inhibition (Cowen and Lindquist 2005). Indeed, fevers may generally exist to compromise an invading pathogen's ability to evolve resistance.

We have also shown that in Aspergillus - a major human pathogen that is separated from C. albicans by one billion years of evolution-resistance to a completely different class of antifungal drugs (the echinocandins) also depends on Hsp90 (Cowen and Lindquist 2005). These experiments demonstrate that Hsp90-mediated mechanisms for acquiring drug resistance are very broadly used by diverse human pathogens.
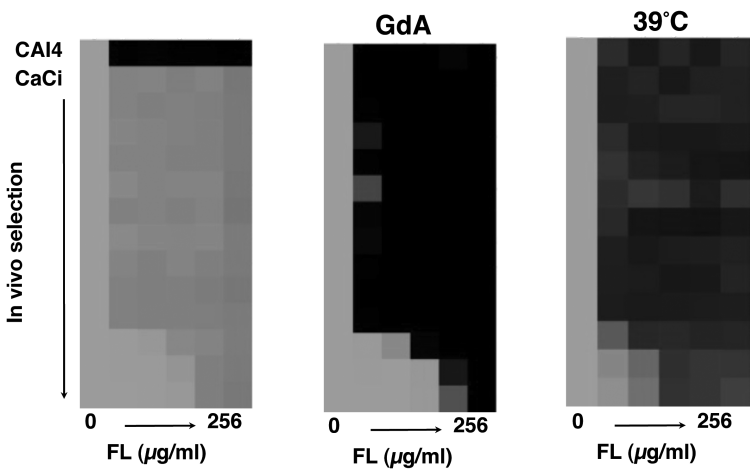

Figure 4. The importance of Hsp90 in fluconazole (FL) resistance in Candida albicans. FL resistance of clinical isolates was initially Hsp90 dependent but evolved toward independence. (Left) FL sensitivity of CAI4 and resistance of serial clinical specimens ( $\mathrm{CaCi}$ ) isolated from an $\mathrm{HIV}$ patient receiving $\mathrm{FL}$; isolates are ordered sequentially, with those recovered early at the top. (Middle) Inhibition of Hsp90 by GdA (5 mM). (Right) Elevated temperatures reduce FL resistance of $\mathrm{CaCi}$ isolates. (Reprinted, with permission, from Cowen and Lindquist 2005 [AAAS].)

\section{HSP90'S EFFECTS ON NATURALLY OCCURRING VARIATION ARE FREQUENTLY BENEFICIAL}

To get a sense of how widespread Hsp90-dependent genetic variation can be, we have now exposed 96 diverse yeast strains from many ecological niches to different growth conditions. This created a wide variety of phenotypes that were readily assayed in the lab. We compared the growth of those cells with and without subjecting them to modest Hsp90 inhibition. We found that Hsp90 inhibition can both uncover and mask traits in a manner that depends on genetic background (Fig. 5). Every trait we studied was affected in at least some of the strains we tested (D Jarosz, unpubl.). These experiments provide evidence for widespread naturally occurring genetic variation, upon which Hsp90 can act. They also establish that Hsp90 acts on this variation both as a potentiator and as a capacitor and, most importantly, that the effects were as often beneficial as detrimental.

Remarkably, these observations provide a plausible mechanism for understanding the rapid evolution of complex traits and suggest that evolution can be driven in unexpected ways by exposure to certain environmental stresses. Moreover, understanding these evolutionary processes will help us to guide new therapeutic strategies for cancer (Dai and Whitesell 2005; Whitesell and Lindquist 2005; Cullinan and Whitesell 2006) and infectious disease (Cowen 2008; Cowen et al. 2009).

\section{PRIONS}

Not only have we shown that Hsp90 and environmental stress influence the interface between genotype and phenotype, but we have also found a completely different way in which protein folding affects this interface: prions (Shorter and Lindquist 2005). Prions produce a vast array of new heritable phenotypes through a heritable, self-per- 

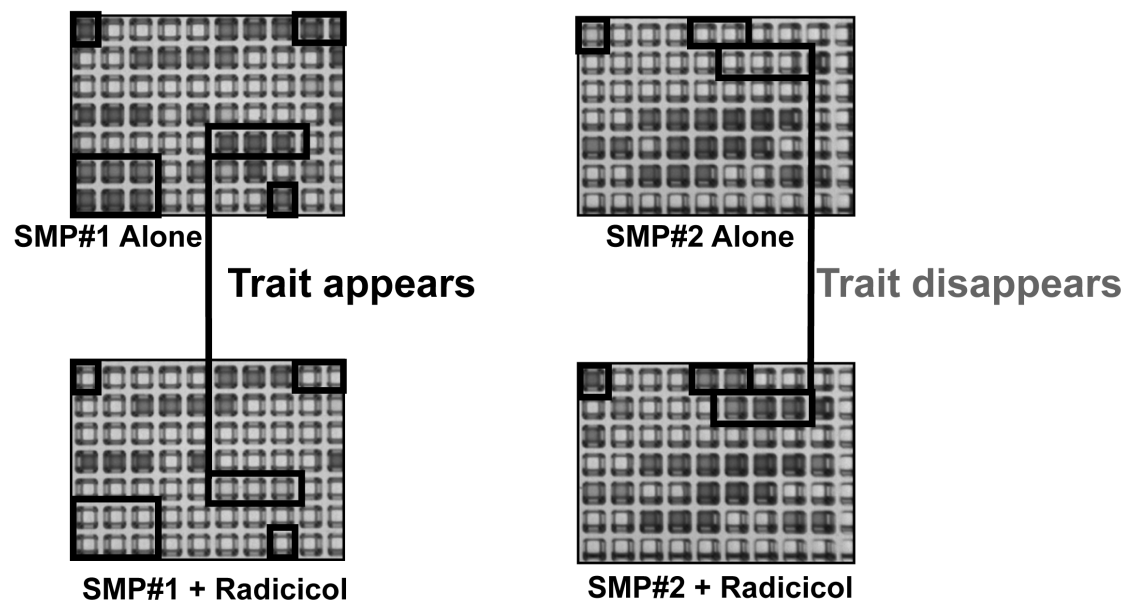

Figure 5. Examples of phenotypic transitions elicited in S. cerevisiae by reducing the Hsp90 buffer. Genotyped meiotic segregants from a cross between two sequenced genetic backgrounds were arrayed in 384-well plates, and their growth was monitored across a broad array of conditions and in parallel when reducing the Hsp90 buffer. Under some conditions, growth is enhanced when the Hsp90 buffer is reduced, and this occurs in segregants with shared genetic polymorphisms. Growth can also be reduced when Hsp90 is inhibited. Again, the response occurs in segregants that share certain regions of their genomes.

petuating change in protein conformation. I will for just one of these elements: $\left[P S I^{+}\right]$.

The $\left[P S I^{+}\right]$prion is formed from an inactive, misfolded translation-termination factor, Sup35, which is a self-templating amyloid. The misfolded proteins are passed from mother cell to daughter and act as "seeds" to perpetuate the alternately folded prion state from generation to generation. This results in the heritable read-through of stop codons throughout the genome. We asked what the general consequences of $\left[\mathrm{PSI}^{+}\right]$might be on yeast biology, by testing seven isogenic $\left[\mathrm{PSI}^{+}\right] /\left[\mathrm{psi}^{-}\right]$strains for growth under 200 conditions (True and Lindquist 2000). Remarkably, in each genetic background, $\left[P S I^{+}\right]$produced a completely different constellation of phenotypes. Thus, $\left[P S I^{+}\right]$rapidly uncovers previously hidden genetic variation in a stepwise fashion. Presumably, it does so by reactivating genes previously inactivated by stop-codon mutations, ribosomal frame-shifting (Namy et al. 2008), uncovering cryptic coding potential in the $3^{\prime}$-untranslated regions of already active genes, and changing the half-lives of messages through well-known mechanisms.

We recently devised high-throughput methods to scan the entire yeast genome and to detect additional candidate prion-coding sequences, identifying $\sim 200$. We tested the top 100 candidates for hallmarks of prion activity: ability to form amyloids, heritability and infectivity, and ability to alter their functional state when their conformational state changes. Applying our set of assays, we found 24 prion candidates that satisfactorily met these criteria. Included in that number were all four of the known yeast prions as well as new prions involved in functions as diverse as cell wall structure and carbon metabolism (Alberti et al. 2009).

Evolutionary change is fueled by genetic diversity and fluctuating environments. The $\left[\mathrm{PSI}^{+}\right]$prion allows cells to express diverse phenotypes in a heritable manner with no change in the genome. It provides a route for selection to act on genetic variants that normally have no phenotypic consequence and to fix them through the reversion of stop codons. This would allow maintenance of the trait when cells return to the normal $\left[\mathrm{psi}^{-}\right]$state. When yeast cells are stressed, switching between the prion and nonprion states occurs at a higher rate (Tyedmers et al. 2008), which may give the yeast a better chance to adapt to challenging conditions. Thus, the link among prion biology, protein folding, and environmental stress seems to create a "bet-hedging" strategy. Just as with alterations in the Hsp90 buffer, the new phenotypes that arise due to prion switching are sometimes beneficial and sometimes detrimental. If a few cells ( 1 in $10,000$ to 1 in 100,000$)$ in the population switch to a detrimental state, it would not matter very much for survival of the population. But if the traits are beneficial, they could matter a great deal. Our work opens several new avenues of study, including the application of similar strategies to find additional prions in yeast, thorough investigation of these prions in vivo and in vitro, and scanning of other organisms' genomes for prion candidates.

\section{ACKNOWLEDGMENTS}

This work was supported by National Institutes of Health grant R01GM025874, a SPARC grant from the Broad Institute, the G. Harold and Leila Y. Mathers Foundation, and the Howard Hughes Medical Institute. I also thank Dr. Karen Allendoerfer for preparing the transcript of the lecture on which this manuscript is based and Drs. Daniel Jarosz and Mikko Taipale for helpful comments on the manuscript.

\section{REFERENCES}

Alberti S, Halfmann R, King O, Kapila A, Lindquist S. 2009. A systematic survey identifies prions and illuminates sequence features of prionogenic proteins. Cell 137: 146-158. 
Cowen LE. 2008. The evolution of fungal drug resistance: Modulating the trajectory from genotype to phenotype. Nat Rev Microbiol 6: 187-198.

Cowen LE, Lindquist SL. 2005. Hsp90 potentiates the rapid evolution of new traits: Drug resistance in diverse fungi. Science 309: 2185-2189.

Cowen LE, Singh SD, Kohler JR, Collins C, Zaas AK, Schell WA, Aziz H, Mylonakis E, Perfect JR, Whitesell L, et al. 2009. Harnessing Hsp90 function as a powerful, broadly effective therapeutic strategy for fungal infectious disease. Proc Natl Acad Sci 106: 2818-2823.

Cruz MC, Goldstein AL, Blankenship JR, Del Poeta M, Davis D, Cardenas ME, Perfect JR, McCusker JH, Heitman J. 2002. Calcineurin is essential for survival during membrane stress in Candida albicans. EMBO J 21: 546-559.

Cullinan SB, Whitesell L. 2006. Heat shock protein 90: A unique chemotherapeutic target. Semin Oncol 33: 457-465.

Dai C, Whitesell L. 2005. HSP90: A rising star on the horizon of anticancer targets. Future Oncol 1: 529-540.

Fox DS, Heitman J. 2002. Good fungi gone bad: The corruption of calcineurin. Bioessays 24: 894-903.

Imai J, Yahara I. 2000. Role of HSP90 in salt stress tolerance via stabilization and regulation of calcineurin. Mol Cell Biol 20: 9262-9270.

Namy O, Galopier A, Martini C, Matsufuji S, Fabret C, Rousset JP. 2008. Epigenetic control of polyamines by the prion [PSI+]. Nat Cell Biol 10: 1069-1075.

Queitsch C, Sangster TA, Lindquist SL. 2002. Hsp90 as a capacitor of phenotypic variation. Nature 417: 618-624.
Rutherford SL, Lindquist SL. 1998. Hsp90 as a capacitor for morphological evolution. Nature 396: 336-342.

Sangster TA, Bahrami A, Wilczek A, Watanabe E, Schellenberg K, McLellan C, Kelley A., Kong SW, Queitsch C, Lindquist SL. 2007. Phenotypic diversity and altered environmental plasticity in Arabidopsis thaliana with reduced Hsp90 levels. PLoS ONE 2: e648.

Sangster TA, Salathia N, Undurraga S, Milo R, Schellenberg K, Lindquist S, Queitsch C. 2008a. HSP90 affects the expression of genetic variation and developmental stability in quantitative traits. Proc Natl Acad Sci 105: 2963-2968.

Sangster TA, Salathia N, Lee HN, Watanabe E, Schellenberg K, Morneau K, Wang H, Undurraga S, Queitsch C, Lindquist S. 2008b. HSP90-buffered genetic variation is common in Arabidopsis thaliana. Proc Natl Acad Sci 105: 2969-2974.

Shorter J, Lindquist SL. 2005. Prions as adaptive conduits of memory and inheritance. Nat Rev Genet 6: 435-450.

True HL, Lindquist SL. 2000. A yeast prion provides a mechanism for genetic variation and phenotypic diversity. Nature 407: 477-483.

Tyedmers J, Madariaga ML, Lindquist S. 2008. Prion switching in response to environmental stress. PLoS Biol 6: e294.

Whitesell L, Lindquist SL. 2005. HSP90 and the chaperoning of cancer. Nat Rev Cancer 5: 761-772.

Xu Y, Lindquist SL. 1993. Heat-shock protein hsp90 governs the activity of pp60v-src kinase. Proc Natl Acad Sci 90: 7074-7078.

Xu Y, Singer MA, Lindquist S. 1999. Maturation of the tyrosine kinase c-src as a kinase and as a substrate depends on the molecular chaperone Hsp90. Proc Natl Acad Sci 96: 109-114. 


\section{$\$_{\mathrm{CSH}}^{\infty}$ Cold Spring Harbor Symposia SYMPOSIA on Quantitative Biology}

\section{Protein Folding Sculpting Evolutionary Change}

S. Lindquist

Cold Spring Harb Symp Quant Biol 2009 74: 103-108 originally published online April 7, 2010 Access the most recent version at doi:10.1101/sqb.2009.74.043

References This article cites 21 articles, 8 of which can be accessed free at: http://symposium.cshlp.org/content/74/103.full.html\#ref-list-1

License

Email Alerting Receive free email alerts when new articles cite this article - sign up in the box at the Service top right corner of the article or click here.

To subscribe to Cold Spring Harbor Symposia on Quantitative Biology go to: http://symposium.cshlp.org/subscriptions 